\title{
Finite-Element Non-conforming $h$-Adaptive Strategy Based on Autonomous Leaves Graph
}

Diego Brandão, Sanderson L. Gonzaga de Oliveira, and Mauricio Kischinhevsky

Instituto de Computação, Universidade Federal Fluminense

Rua Passo da Pátria, 156, Bloco E, São Domingos, 24210-240, Niterói - RJ - Brazil

http://www.ic.uff.br

\begin{abstract}
Adaptive mesh refinement techniques are used in order to decrease the computational cost associated with the numerical solution of Partial Differential Equations. In this work, the refined mesh is represented by a graph data structure. More precisely. this scheme follows the Autonomous Leaves Graph concepts. The objective is to construct an adaptive mesh refinement with lower cost than tree-based schemes. Moreover, the Autonomous Leaves Graph was initially proposed with the Finite Volume Method and a Modified Hilbert Curve was used for the total-ordering of the control volumes. This work proposes to integrate the Autonomous Leaves Graph and the Finite Element Method as well as to adapt the Modified Hilbert Curve for this scheme. Furthermore, a non-conforming $h$-adaptive strategy is implemented. This approach is applied in the solution of the Poisson equation problem and the experimental results are discussed.
\end{abstract}

Keywords: Finite Element Method, $h$-adaptativity, Autonomous Leaves Graph, Space-filling curves, Numerical Methods, Hilbert Curve, Non-conforming meshes.

\section{Introduction}

Adaptive mesh refinement (AMR) strategies are important in several areas of engineering and uniform meshes have been widely used in order to solve such problems. However, uniform meshes are not a computationally viable choice for solving a system of Partial Differential Equation (PDEs) in which steep gradients, singularities, or discontinuities need to be captured. Thus, since a tree-based structure provides a simple scheme for $h$ and $p$-refinement, it has been broadly used. In this structure, the parents and the refined children elements are represented in the same computational data structure, generating an overhead when scanning the neighbors elements in order to assemble the resulting linear system.

The research in this field had a relevant advance when the Autonomous Leaves Graph (ALG) was proposed for the Finite Volume Method and a space-filling curve, named Modified Hilbert Curve (MHC), was proposed in order to number the control volumes [1]. This present work proposes the ALG technique for the Finite Element Method (FEM) and an adaptation of the MHC for this scheme as well.

G. Allen et al. (Eds.): ICCS 2009, Part I, LNCS 5544, pp. 570 579, 2009.

(C) Springer-Verlag Berlin Heidelberg 2009 
In the following section, the ALG is briefly presented. Next, Section 3 describes the MHC adaptation to FEM-ALG. Afterwards, Section 4 shows the numerical results. Finally, Section 5 draws some concluding remarks.

\section{Data Structure}

Consider a bidimensional domain in a unit square $\Omega=[0,1]^{2}$. This square is discretized into four new equal ones, obtaining four square discrete places. The discretized domain is represented in Fig. 1.

The graph of Fig. 1 shows black circles, which are called here as black nodes. Black nodes represent the discrete places of the discretized domain in Fig 1. In

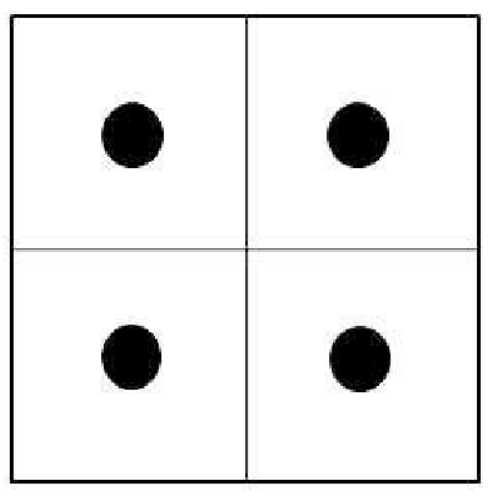

a)

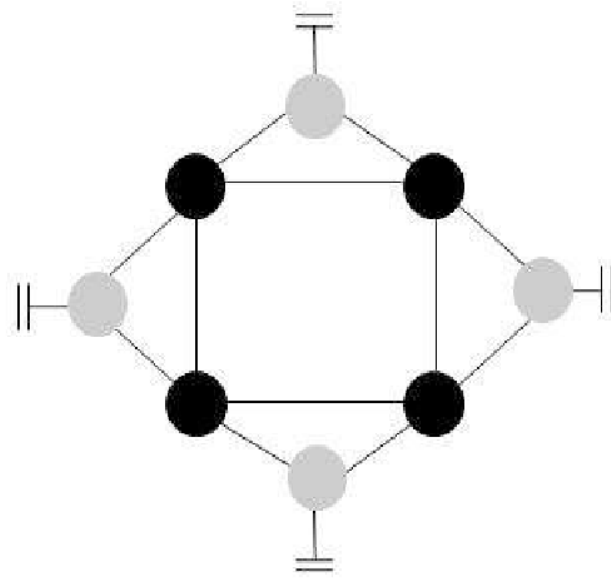

b)

Fig. 1. An initial discretized domain and a graph that represents it
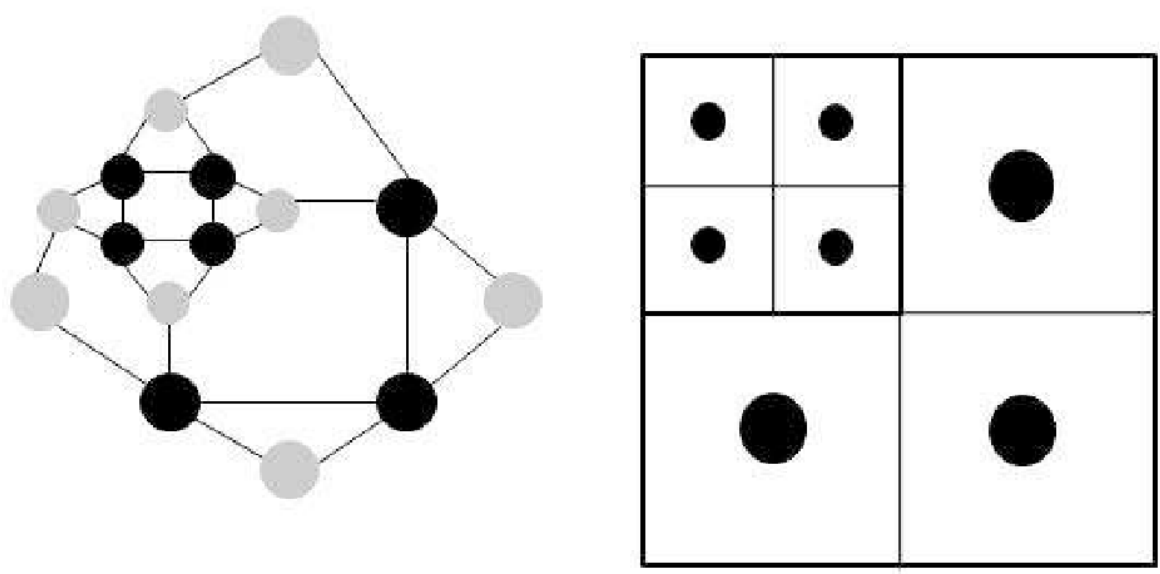

Fig. 2. Refinement process 
addition, the gray circles of the graph represent transition nodes. They furnish connectivity information between nodes with different level of refinements. The ones in Fig. 1 represent connectivity information within the boundary of the domain.

In the refinement process, an element in a level $n$ is replaced by four black nodes and four transition nodes in a level $n+1$. This process is sketched in Fig. 2

\section{MHC Adaptation}

The ALG was proposed as a graph data structure in which each node represents a control volume. Similarly, this present work proposes a graph data structure in which each node represents a finite element.

Since non-conforming meshes are not represented by the Hilbert Curve, the original ALG proposed the MHC in order to number the barycenter of the control volumes. The $\mathrm{MHC}$ was implemented by a linked list. Likewise, this present work implements two linked lists in order to number: $i$ ) the barycenter of the finite elements (likewise the original ALG)), i.e. a linked list to number the finite elements and ii) the vertices of the non-conforming finite elements. Moreover, since in the FEM the values are evaluated in the element vertices, the second linked list is an adaptation of the MHC algorithm in order to number the vertices of the finite elements. Figure 3 depicts an example of the vertice numbering by the adaptation of the MHC.

Each node of the second linked list represents a mesh vertex as well as retains relevant information about which finite element it belongs to. Furthermore, each

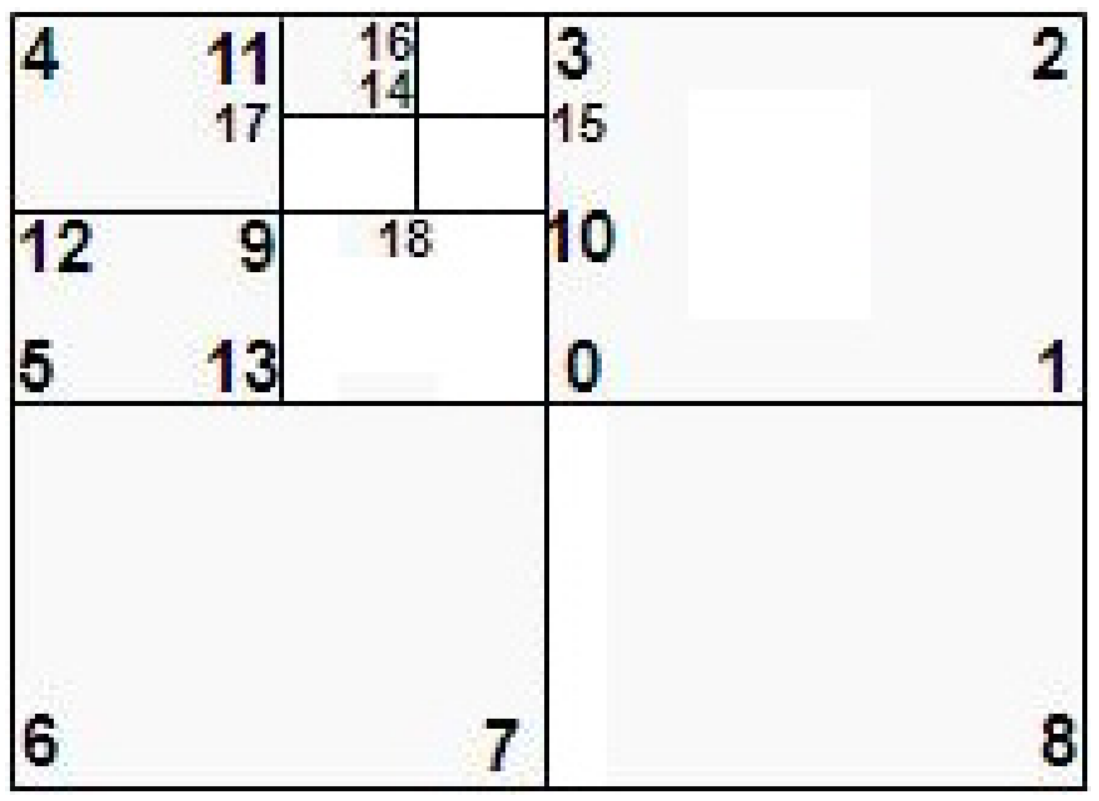

Fig. 3. Example of the vertice numbering by the adaptation of the MHC 
node of the second linked list is allocated for this purpose whereas the pointers of the first linked list (which numbers the proper finite elements) are included in the proper graph nodes. In other words, there is a pointer in each graph node and these pointers implement the (first one) linked list to number the finite elements.

\subsection{Assembling the Resulting Stiffness Matrix}

The goal of those structures is to assemble the resulting stiffness matrix with the adequate connectivity information among neighbor elements. Furthermore, each line of the stiffness matrix is associated with a mesh vertice and is represented by a different simple linked list. In addition, since this scheme produces a sparse stiffness matrix, only non-zero values as well as their position are saved into the linked lists that represent the stiffness-matrix lines.

Notice that there are a linked list to number the finite elements, a linked list to number the vertices and each line of the resulting stiffness matrix is also a linked list.

Those linked lists that represent the stiffness-matrix lines are updated by a computational procedure that scans the vertex-linked list. It applies a linear interpolation of the associated corner-element values in order to solve the hanging nodes.

Numerical results show that this numbering scheme produces a sparse symmetric positive-definite matrix [2]. Moreover, the Conjugate Gradient Method was used in order to solve the resulting stiffness matrix.

\section{Numerical Results}

Firstly, results of two patch tests of the Laplace Problem is presented. Besides, the rule 2:1 was used in order to control the number of refinements. Secondly, a Poisson equation problem that involves steep gradients is solved. A $4 \times 4$ GaussLegendre Quadrature on four-node bilinear quadrilateral elements was applied. In the numerical tests, the maximum permissible error in the energy norm was set to five percent $\left(\eta_{\max }=0.05\right)$.

\subsection{AMR Strategy}

Consider the elliptic boundary-value problem

$$
-\nabla^{2} u(x)=f(x) \text { in } \Omega,
$$

subject to the boundary conditions

$$
u=0 \text { on } \partial \Omega .
$$


The variational form of these equations can be written in the bilinear form

$$
B(u, v)=\int \nabla u \nabla v d \Omega
$$

where $v \in H_{0}^{1}(\Omega)$.

Observe that $H_{0}^{1}(\Omega)$ is the Sobolev Space of functions with square-integrable derivatives and the values on $\partial \Omega$ vanish.

One can define the error as

$$
\text { error }=u-u_{h},
$$

where $u$ is the real solution and $u_{h}$ is the numerical approximation. However, since this local measure is not computationally convenient, mathematical norms are introduced in order to measure the error. The energy error norm is related to the weak form in Eq. (3), thus,

$$
\|e\|^{2}=B(e, e)=\int \nabla e \nabla e d \Omega .
$$

Since the exact solution field is generally unknown, the approximate solution is post-processed in order to obtain a more accurate measure for the gradient of $u_{h}[3]$. The relative percentage error in the energy norm is

$$
\eta=\frac{\|e\|}{\|u\|}
$$

where $\|u\|$ is the exact energy norm.

A simple criterion in order to achieve a solution with an acceptable error is

$$
\eta_{\max } \leq \eta,
$$

where $\eta_{\max }$ is the maximum permissible error percentage in the whole domain and

$$
\eta=\frac{\|e\|}{\left(\left\|u^{h}\right\|^{2}+\|e\|^{2}\right)^{\frac{1}{2}}} .
$$

The error should be equally distributed among the elements in order to obtain an optimal mesh

$$
\|e\|=\sqrt{m}\|e\|_{i},
$$

where $m$ is the number of elements.

The error can be expressed for the entire domain using the admissible elementar error [4] 


$$
\|e\|_{i} \leq \eta_{\max }\left(\frac{\left(\left\|u^{h}\right\|^{2}+\|e\|^{2}\right)}{m}\right)^{\frac{1}{2}} \equiv e_{m}^{-} .
$$

Furthermore, if the error in an element is

$$
\xi_{i}=\frac{\|e\|_{i}}{e_{m}^{-}}>1
$$

the error in the element $i$ is larger then the criterion and, therefore, it needs to be refined.

\subsection{Validation}

The Laplace problem

$$
\nabla^{2} u=0, \Omega=[0,1]^{2},
$$

with $u=g(x)=x_{1}+x_{2}$ over $\partial \Omega$ is solved.

The analyical solution is $u(x)=x_{1}+x_{2}$. In this test, $\eta_{\max }=0.0$ was adopted in order to refine the elements. Figure 4 depicts an example of the accuracy of this test.

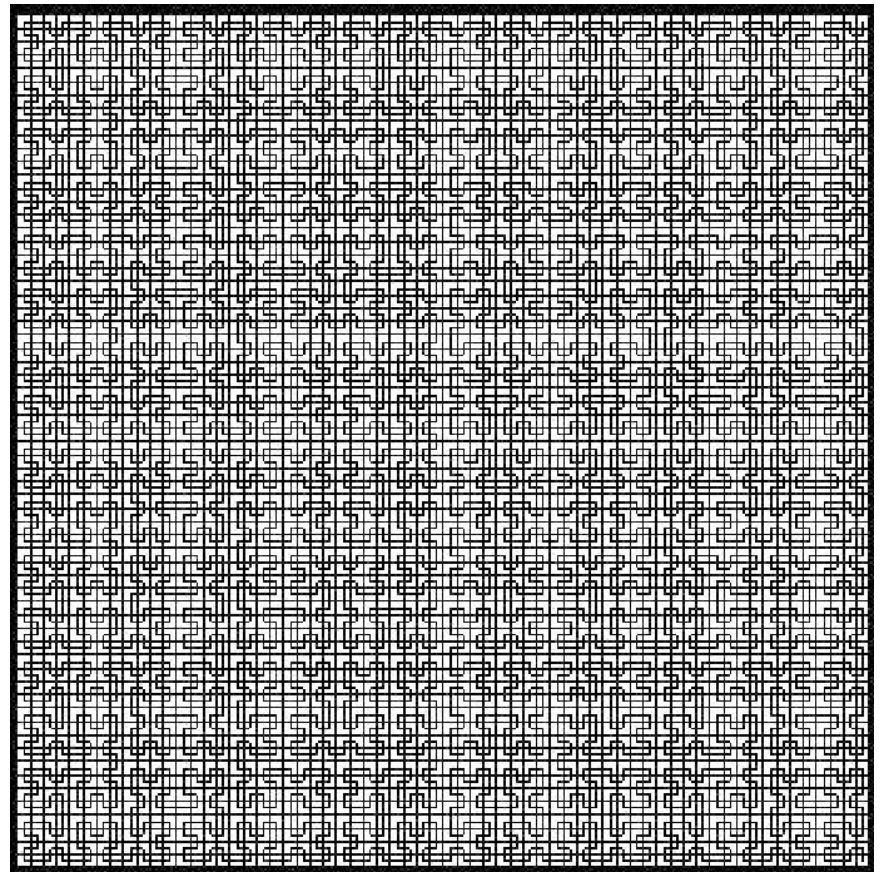

Fig. 4. Uniform refinement of the Laplace Problem comprising 1089 vertices 
Table 1. Table 1: Laplace Problem results

\begin{tabular}{|c|c|c|c|}
\hline Mesh & Number of elements & Number of vertices & Energy norm error \\
\hline $\mathrm{a}$ & 16 & 25 & $1.7 \times 10^{-17}$ \\
$\mathrm{~b}$ & 256 & 289 & $4.4 X 10^{-18}$ \\
$\mathrm{c}$ & 1024 & 1089 & $2.2 \times 10^{-18}$ \\
\hline
\end{tabular}

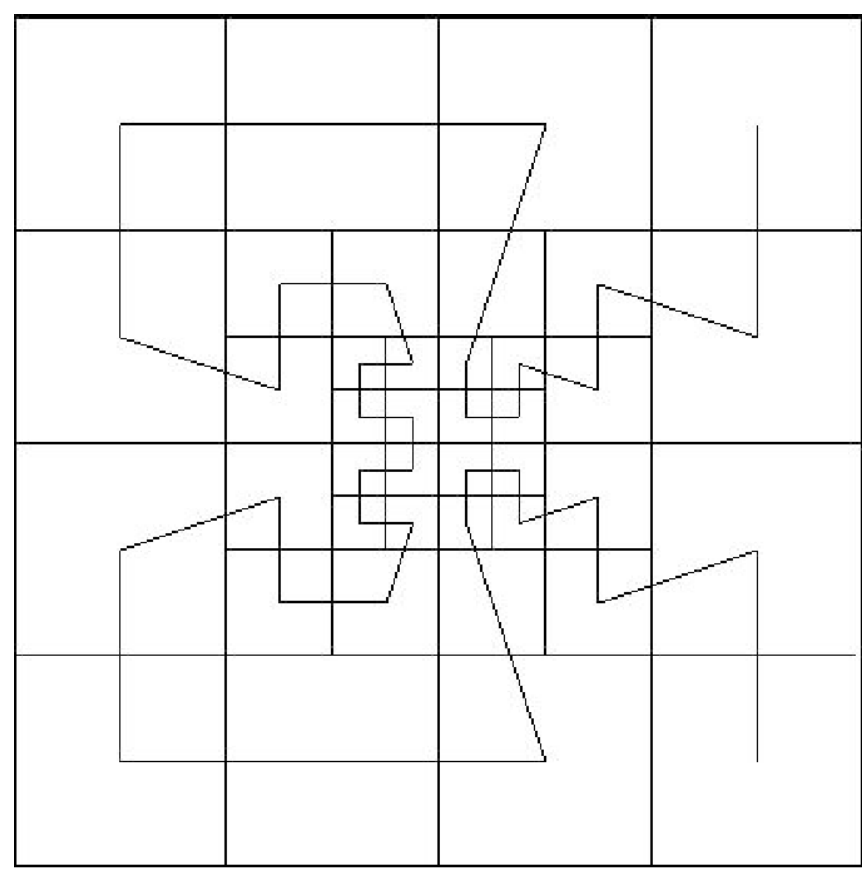

Fig. 5. Mesh of the Laplace Problem with 41 vertices and the associated MHC

Table 1 shows a comparison of the energy norm error among different meshes. A better numerical approximation when more elements are in the mesh is obtained.

Another test admitted 0.05 as the maximum error $\left(\eta_{\max }\right)$. Figure 5 shows an example mesh for this test. Fig. 6 presents a graphic of the vertice quantity by the error of the energy semi-norm $H_{1}$, showing the convergence of the solution.

\subsection{Test in the Poisson Equation Problem}

Consider the Poisson equation problem in $\Omega=[0,1]^{2}$ with Dirichlet boundary conditions

$$
-\nabla^{2} u=f(x, y)
$$




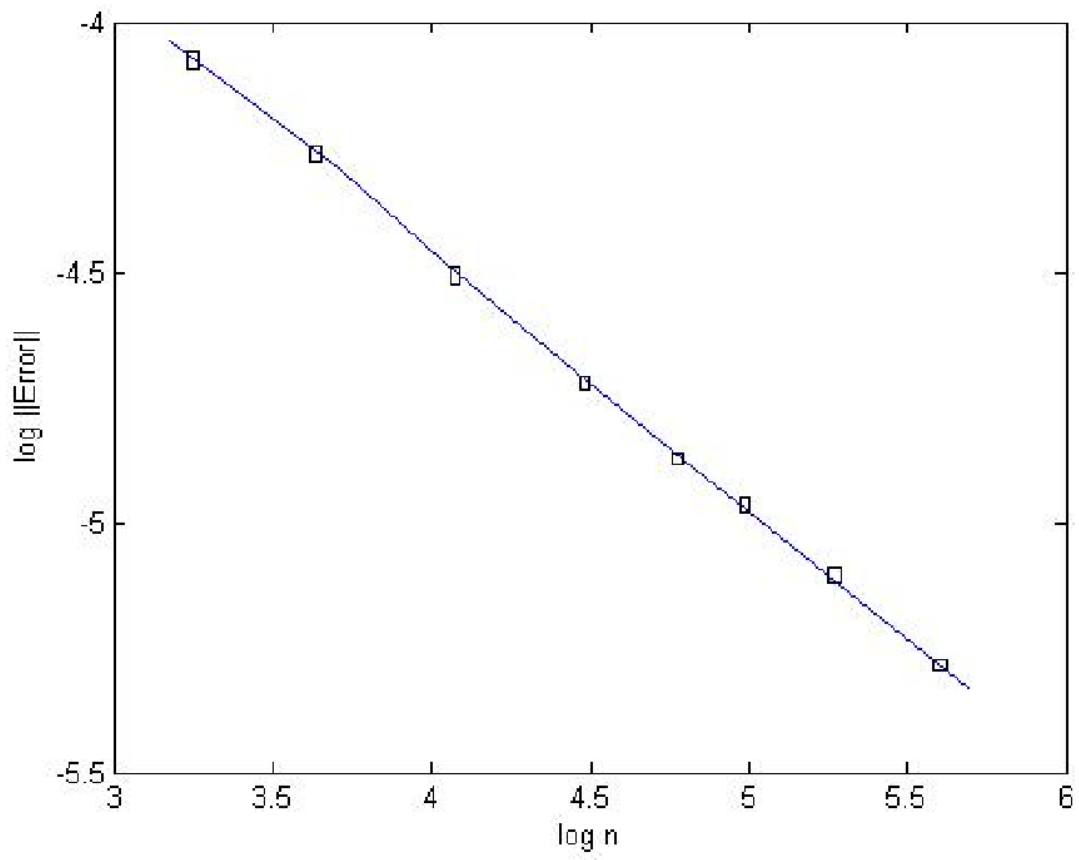

Fig. 6. Refinament convergence rate where $n$ is the number of equations

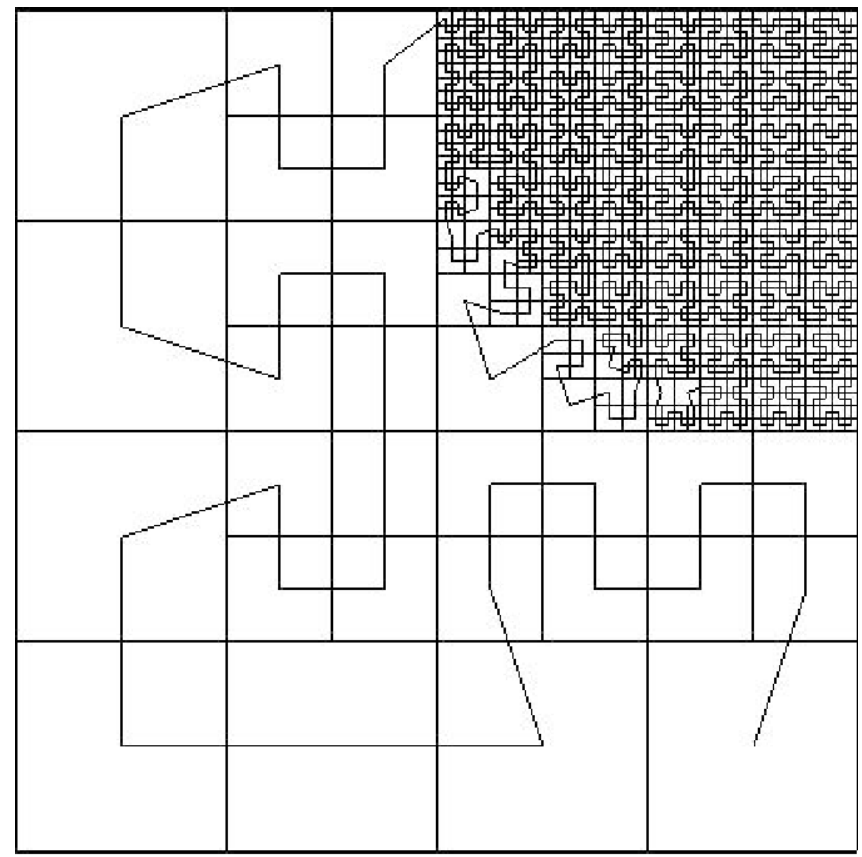

Fig. 7. Mesh of the Poisson equation problem with 248 elements 


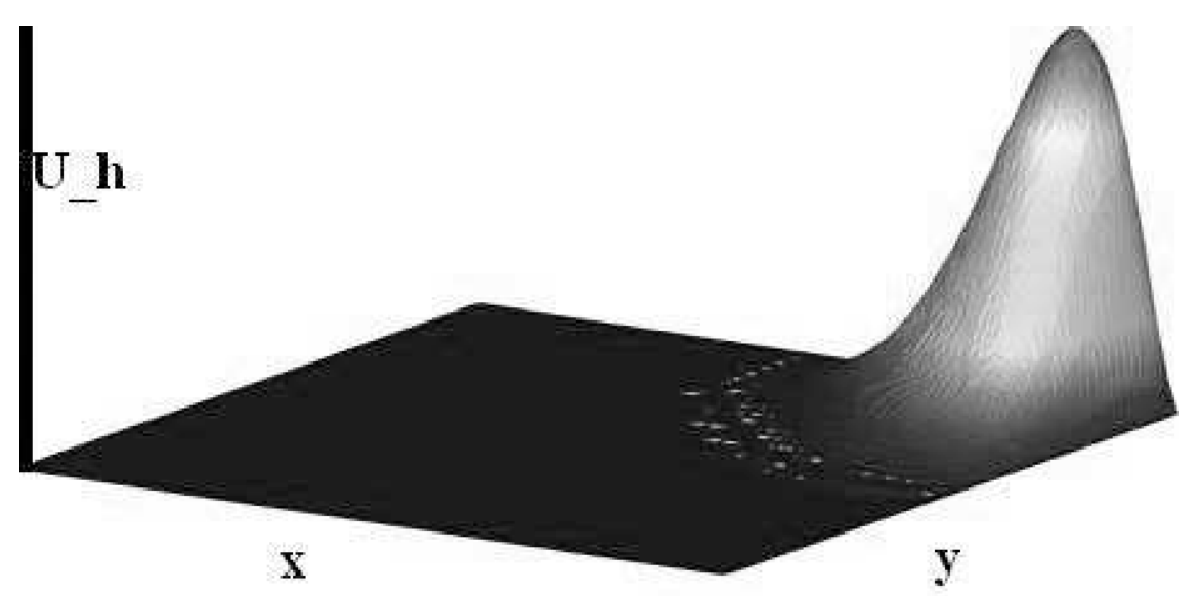

Fig. 8. Behavior of the solution $u_{h}$ for the Poisson equation problem

with condition $u=0$ over $\partial \Omega$.

The source term is given by

$$
\begin{array}{r}
f(x, y)=-90 x^{8} y^{10}(1-x)(1-y)+20 x^{9} y^{10}(1-y)- \\
90 x^{10} y^{8}(1-x)(1-y)+20 y^{9} x^{10}(1-x) .
\end{array}
$$

The analitical solution is $u(x)=x^{10} y^{10}(1-x)(1-y)$. Figure 7 shows a mesh for this test. Figure 8 shows that FEM-ALG represents the gradient close to the point $(1,1)$.

\section{Concluding Remarks}

FEM-ALG is a novel technique for the adaptive mesh refinement that integrates the FEM and the ALG. This scheme intends to be more computationally efficient than other AMR schemes when searching for connectivity information among elements with different levels of refinement since the refinement process is merely local when updating all the involved structures.

This work shows experimental results of this scheme in the Poisson equation problem. More experiments shall be performed in the heating conduction equation and in the wave equation in future works. Moreover, future works will describe the unrefinement process. Besides, future works will describe the integration of new techniques in order to treat hanging nodes as well as the integration of mesh-free methods in order to apply the shape functions on nonconforming meshes. 


\section{Acknowledgements}

The authors gratefully acknowledge CNPq, CAPES and FAPERJ for the financial support.

\section{References}

1. Burgarelli, D., Kischinhevsky, M., Biezuner, R.: A new adaptive mesh refinement strategy for numerically solving evolutionary PDE's. J. of Computational and Applied Mathematics. 196, 115-131 (2006)

2. Brandao, D.: A h-adaptative mesh refinement for the Finite Element Method using a graph structure (in Portuguese). Instituto de Computação da Universidade Federal Fluminense (2008)

3. Tabarraei, A., Sukumar, N.: Adaptive computations on conforming quadtree meshes. Finite Elements in Analysis and Design 41, 686-702 (2005)

4. Hughes, T.: The Finite Element Method. Englewood Cliffs (1987) 